\title{
Does the distribution of proerectile nerves support the use of a high anterior incision?
}

A team of researchers from Germany, led by Roman Ganzer, has examined the topographical distribution of nerves around the prostate in order to clarify which regions are important for erectile function. "Our intention was to use immunohistochemistry to distinguish proerectile parasympathetic nerves from nonerectile sympathetic fibers," says Ganzer.

Improved understanding of nerve distribution around the prostate is crucial for the continued development of nervesparing prostatectomy techniques and improvements in potency rates. Previous studies have shown that, rather than being exclusively clustered in the dorsolateral neurovascular bundle, nerve fibers are also present in the ventrolateral portion of the prostate. This observation led to the adoption of a high anterior incision for surgery to ensure preservation of these nerves; however, it was not previously known whether ventrolateral nerves actually contribute to erectile function.

\section{4 ...nerve distribution was} variable in the ventrolateral region... 77

To answer this question, investigators took whole mount sections of the prostates from 49 patients who underwent nonnerve-sparing radical prostatectomy, and excluded any specimens that had incomplete prostatic capsules or periprostatic tissue. Five consecutive sections $(10 \mu \mathrm{m})$ were taken from the base, the middle and the apex of each prostate $(n=38)$. One section was stained with hematoxylin and eosin, two were stained with an antibody against tyrosine hydroxylase to identify sympathetic

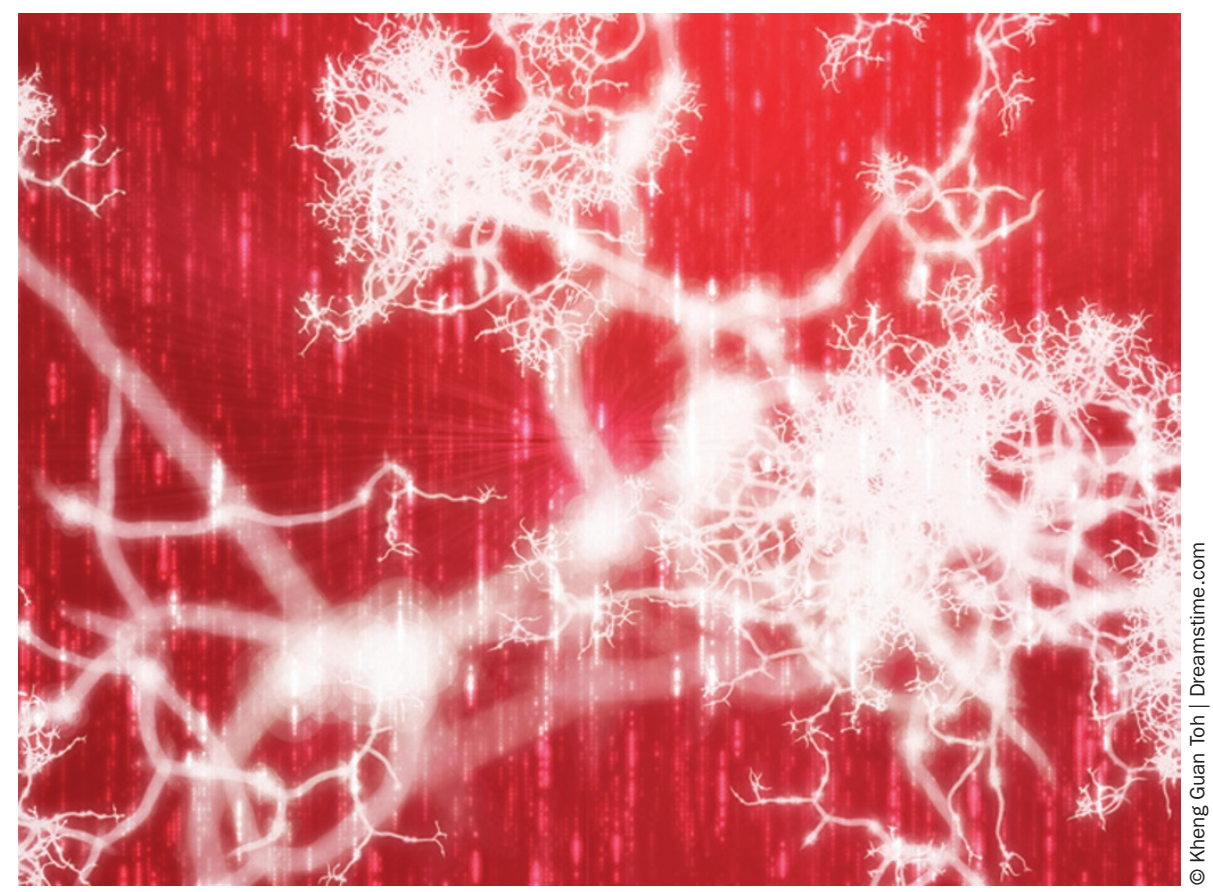

fibers, and two were stained with an antibody against vesicular acetylcholine transporter to mark parasympathetic nerves. Researchers then counted the parasympathetic and sympathetic nerves in each region (ventrolateral versus dorsolateral) and calculated mean values from two adjacent sections.

In total, 999 parasympathetic and 1,531 sympathetic fibers were counted, and the majority of both were found in the dorsolateral part of the prostate, presumably corresponding to the neurovascular bundle. However, nerve distribution was variable in the ventrolateral region. Proerectile parasympathetic nerves were more dispersed at the base than the apex, with $12.4 \%$ and $1.5 \%$ of nerves located ventrolaterally, respectively, while the proportion of sympathetic nerves in the ventrolateral region remained constant (6-9\%). In other words, says Ganzer, "despite the presence of ventrolateral periprostatic nerves, only a minority of these seem to have parasympathetic proerectile quality."

Although this might suggest that the high anterior incision is unnecessary, Ganzer points out there might be other reasons for its use, including reduced traction and improved anatomical support of the neural structures.

Sarah Payton

Original article Ganzer, R. et al. Anatomic study of periprostatic nerve distribution: immunohistochemical differentiation of parasympathetic and sympathetic nerve fibres. Eur. Urol. doi:10.1016/j.eururo.2012.03.039 